\title{
P05.16. Subjects' experiences of a bi-weekly yoga and Ayurveda-based weight loss protocol in overweight and obese women
}

\author{
T Braun $^{1 *}$, L Conboy ${ }^{2}$ \\ From International Research Congress on Integrative Medicine and Health 2012 \\ Portland, Oregon, USA. 15-18 May 2012
}

\section{Purpose}

Yoga is one of the most common forms of complementary and alternative therapies utilized for weight loss. This mixed methods study included quantitative surveys and interviews of participants of a 10-week, bi-weekly yoga and Ayurveda-based weight loss program designed for overweight and obese women. The program involved training in yoga poses, breathing, and philosophy as well as eating mindfully, cultivating relaxation, and compassion.

\section{Methods}

This analysis focuses on the subject interviews which occurred both pre and post program and covered the topics of: (1) hopes and found benefits of the program, (2) anticipated challenges and lessons learned, (3) subjects' relationships with food pre and post program. Analysis was directed by a grounded theory approach. The interview notes were double coded independently by the two authors. Any disagreements in coding were addressed in discussion. Coding consisted of searching for sought themes and emergent themes in each transcript as well as condensing responses to each topic across the sample.

\section{Results}

Thirty-seven overweight and obese participants were enrolled (mean BMI of 34.1; SD +/-6.1) with mean age of 48.3 years. Most were yoga naïve and had tried weight loss programs in the past. The qualitative findings support the trial's quantitative findings of statistically significant improvements baseline to post program in BMI $(\mathrm{p}<0.05)$, and a number of psychosocial variables. Pre-program, subjects reported that they were drawn to the yoga-based program to achieve greater clarity, structure, and security in their lives in addition to losing weight. Post program, subjects reported greater sense of security, self-confidence, self-compassion, and insight into their behavioral motivations. Useful program tools mentioned are: breath work, mindful eating, focus on self-care, relaxation.

\section{Conclusion}

Our data suggests that participation in a 10-week yoga and Ayurveda-based weight loss program is associated with improvements in reported psychosocial health immediately following program completion.

\section{Author details}

'Kripalu Center for Yoga and Health, Lenox, USA. ${ }^{2}$ Harvard Medical School Osher Research Center, Boston, USA.

Published: 12 June 2012

doi:10.1186/1472-6882-12-S1-P376

Cite this article as: Braun and Conboy: P05.16. Subjects' experiences of a bi-weekly yoga and Ayurveda-based weight loss protocol in overweight and obese women. BMC Complementary and Alternative Medicine 2012 12(Suppl 1):P376.

${ }^{1}$ Kripalu Center for Yoga and Health, Lenox, USA

Full list of author information is available at the end of the article

(c) 2012 Braun and Conboy; licensee BioMed Central Ltd. This is an Open Access article distributed under the terms of the Creative 